\title{
Back to the Future!
}

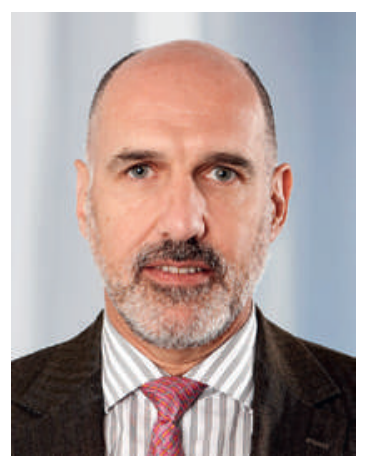

$\mathrm{Au}$ cours des prochaines années, le corps médical, qui a déjà beaucoup dû évoluer, va encore se trouver confronté à des changements marquants: évolution scientifique, vieillissement de la population, pression sur les coûts et le financement, technologies de l'information.

Le Comité central, au moment de tirer le bilan de sa législature, s'est également penché sur le futur, en préparant les «Objectifs politiques et stratégiques» qu'il présentera à la Chambre médicale du 7 juin prochain.

La FMH est l'organisation professionnelle faîtière des médecins suisses; pour avoir le poids nécessaire à défendre les intérêts de ses membres et à les soutenir dans leur pratique de la médecine, la FMH doit renforcer encore sa position de partenaire solide et crédible du système de santé. Il est clair que pour cela, le corps médical doit être aussi uni que possible, et cohérent dans son expression.

\section{Ce programme demandera un engagement soutenu de nos membres et de tous leurs représentants, et des structures solides qui permettent la cohérence nécessaire.}

Cela suppose des structures assurant la cohésion des médecins, dans le dialogue et le respect des spécificités professionnelles et régionales.

En nous appuyant sur ces structures, il nous faudra, d'ici 2016, terme de la législature à venir, continuer d'affirmer notre présence politique et augmenter notre capacité d'intervention, pour que les conditions-cadres de l'exercice de notre profession s'améliorent.

Concrètement, cela va s'exprimer dans une quantité de domaines différents.

Sur le plan tarifaire, pour commencer. Le projet de révision du TARMED doit être mené à bien et mis en œuvre, avec bien sûr son nouveau chapitre pour les médecins de premier recours, et bien sûr aussi sans ponctionner les tarifs des spécialistes, contrairement à ce qu'on continue à entendre de la part des caisses-maladie. C'est le domaine par excellence dans lequel la cohésion du corps médical est essentielle, car la moindre divergence publique entre groupes de médecins aurait des conséquences dramatiques.

L'informatisation de la médecine, ensuite, est un domaine qui va encore croître en importance; outre les questions techniques qui restent à régler, cela supposera des investissements considérables dans les cabinets, qu'il n'est pas question que les médecins assument alors que ces coûts ne sont pas prévus dans les tarifs - le financement devra donc être prévu lors de chaque modification législative.

Et la préservation du secret médical est un point sur lequel la FMH devra continuer à être particulièrement active. La possession des données revêt une importance croissante et la pression augmente en conséquence: l'intérêt et les droits des patient-es doivent continuer à primer, et nous nous battrons pour cela!

La lutte contre la pénurie de médecins se heurte au fédéralisme, et la FMH devra trouver là des canaux d'intervention qui lui permettent d'agir quand même, car le problème est de plus en plus aigu, malheureusement.

Les exemples de la manière dont la FMH va continuer à s'affirmer et à développer son rôle pour garantir et améliorer notre cadre de travail sont nombreux; dans le cadre de cet éditorial, je dois me limiter à citer encore la prévention, et notamment le financement des prestations de prévention fournies par les médecins; et tout le domaine de la qualité, où la question du financement de nos activités se pose également, ainsi que celle de la place et du rôle de ce qu'on appelle le Health Technology Assessment, l'évaluation du rapport coût/bénéfice des prestations.

On le voit, le «programme» préparé par le Comité central pour la prochaine législature est vaste: c'est bien le contexte politique dans lequel le système de santé se développe, dans lequel la médecine s'exerce, qui l'exige.

Ce programme demandera un engagement soutenu de nos membres et de tous leurs représentants, et des structures solides qui permettent la cohérence nécessaire - c'est tout ce que l'on peut souhaiter à la FMH!

Dr Jacques de Haller, Président de la FMH

\section{Le rapport de gestion 2011 est arrivé}

Ecrit dans un style agréable et conçu comme un magazine, il passe non seulement en revue les principaux événements de l'année écoulée, mais présente clairement les principaux points de vue et positions du corps médical sur différents thèmes. L'Institut suisse pour la formation postgraduée et continue (ISFM) informe de ses activités et projets pour l'année 2011 dans son propre rapport de gestion. Pour télécharger et consulter ces documents, veuillez suivre les liens suivants: www.fmh.ch $\rightarrow$ FMH $\rightarrow$ Rapport de gestion ou www.siwf.ch $\rightarrow$ ISFM Bases $\rightarrow$ Rapports de gestion 